\title{
9-Acridinemethanamine and Acridine-9-Carboxaldehyde as Potential Fluorescence Lifetime pH Indicators
}

\author{
Christian Totland $^{1,2}$ (D) Peter J. Thomas ${ }^{3} \cdot$ Bodil Holst $^{4} \cdot$ Naureen Akhtar $^{4} \cdot$ Jostein Hovdenes $^{5} \cdot$ Tore Skodvin $^{1}$
}

Received: 24 February 2020 / Accepted: 25 May 2020 / Published online: 4 June 2020

(C) The Author(s) 2020

\begin{abstract}
A significant challenge concerning the development of fluorescence lifetime (FL) based $\mathrm{pH}$ sensors is the paucity of fluorophores with sufficiently large FL variation with $\mathrm{pH}$. Acridine is amongst the indicators with highest fluoresce lifetime responses to $\mathrm{pH}$, with a change in lifetime of about $13 \mathrm{~ns}$ within a $\mathrm{pH}$ range of 5-8. Here we examine the two acridine derivatives, 9acridinemethanamine (9-AMA) and acridine-9-carbaldehyde (9-ACA) in terms of their FL pH sensitivity and pH sensing range. Both indicators are characterized when dissolved in buffer solutions, as well as when immobilized in support materials. 9-AMA

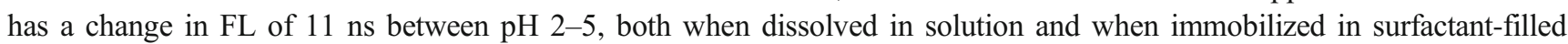
mesoporous silica. The FL of 9-ACA is not sensitive to $\mathrm{pH}$ when dissolved in buffer solutions; however, when covalently bound to amine-modified silica, its FL changes 15 ns between $\mathrm{pH}$ 3-6. 9-AMA and 9-ACA represent promising FL in the $\mathrm{pH}$ range of $\mathrm{pH} 2-6$, and could potentially form the basis of new FL pH sensors.
\end{abstract}

Keywords pH sensor $\cdot$ Fluorescence lifetime $\cdot$ Acridine $\cdot$ 9-acridinemethanamine $\cdot$ Acridine-9-carbaldehyde

\section{Introduction}

In recent years, the research and development of optical chemical sensor technology has been increasing due to their low cost, low power consumption and good long-term stability [1]. Such characteristics make optical sensors particularly interesting for applications in oceanography [2], aquaculture [3] and biomedicine [4] amongst other applications. Optical pH sensor technologies in particular are attracting a lot of interest not only because of the widespread importance of $\mathrm{pH}$

Christian Totland

christian.totland@ngi.no

1 Department of Chemistry, University of Bergen, Allégaten 41, 5007 Bergen, Norway

2 Present address: NGI - Norwegian Geotechnical Institute, Sognsveien 72, 0806 Oslo, Norway

3 NORCE Norwegian Research Centre AS, Fantoftvegen 38, 5072 Bergen, Norway

4 Department of Physics and Technology, University of Bergen, Allégaten 55, 5007 Bergen, Norway

5 Aanderaa - a Xylem brand, Sanddalsringen 5b, N-5225 Nesttun, Norway measurements, but also because of the poor long-term stability of commonly available technology such as electrochemical $\mathrm{pH}$ probes. Optical $\mathrm{pH}$ sensors typically comprise a sensing film consisting of a $\mathrm{pH}$ indicator immobilized within an ion penetrable medium. When the $\mathrm{pH}$ of the liquid surrounding the film changes, this causes some optical property of the indicator to change, which can be quantified using low cost and robust optoelectronic methods. Some common challenges facing optical $\mathrm{pH}$ sensors include photo bleaching from sunlight or probe light from the sensor itself, leaching of the indicator from the immobilizing medium and noise contributions from background luminescence, and probe light intensity variations [5]. Fluorescence lifetime (FL) on the other hand is an intrinsic property of the molecule, and is therefore not affected by these factors.

A significant challenge facing the development of FLbased $\mathrm{pH}$ sensors is that very few fluorophores have been identified that exhibit a large change in FL with $\mathrm{pH}$. Sensor architectures revolving around the use of sensor films containing multiple chemical species have been proposed to get around this problem. For example, the dual-lifetime referencing (DLR) method involves a $\mathrm{pH}$ sensitive fluorophore together with a long-lived luminescent reference, where the fluorescence intensity of the $\mathrm{pH}$ sensitive indicator varies with $\mathrm{pH}$. The combined FL of the indicator pair is much 
longer and varies over a wider range than the $\mathrm{pH}$ sensitive indicator alone making the optoelectronic readout of the FL less challenging [6-8]. Other approaches exploit Föster energy transfers, where a $\mathrm{pH}$ insensitive donor with a long luminescence lifetime, such as lanthanide ions, transfers energy to a pH sensitive dye [9-11]. The antenna-mediated effects can cause large changes in lifetime, and thereby has potential for highly accurate $\mathrm{pH}$ sensors. However, some sensors with multiple indicators have the disadvantage that the overall measured FL will be influenced if the fluorescence properties of one indicator changes differently to the other (e.g. due to difference in photo bleaching or leaching behavior). For these reasons, a sensor architecture incorporating a single indicator with sufficiently large $\mathrm{FL} \mathrm{pH}$ variation remains in principle a more attractive option for obtaining long-term calibration stability [12].

Most pH FL indicators, such as fluorescein or fluorescent proteins used for FL imaging [13,14], only have a 1-2 ns change in FL between high and low $\mathrm{pH}$. This is adequate for lab instruments capable of making high precision FL measurements, but insufficient for achieving a reasonable $\mathrm{pH}$ measurement precision in a field deployable $\mathrm{pH}$ sensor, where typically only low cost and compact optoelectronics can be used for performing the lifetime measurements. There has been a lot of effort focused towards finding alternative indicators that show higher FL responses to $\mathrm{pH}$ change. For example, mercaptopropionic acid-capped quantum dots have been shown to exhibit a FL shift from $8.7 \mathrm{~ns}$ to $15.4 \mathrm{~ns}$ in the $\mathrm{pH}$ range $\sim 5$ to 8 [15]. In another case, the FL of carbon dots was demonstrated to shift from $\sim 13 \mathrm{~ns}$ to $19.5 \mathrm{~ns}$ in the $\mathrm{pH}$ range 3.4 to 11 [16]. Recently, diazaoxatriangulenium based $\mathrm{pH}$ indicators exhibiting a lifetime change from $\sim 7$ to $13 \mathrm{~ns}$ were reported over the $\mathrm{pH}$ range 3 to 8 [17].

Acridine is amongst the indicators with highest fluorescence lifetime responses to $\mathrm{pH}$ [18-20]. Acridine derivatives are much studied for their potential applications in cancer treatment [21], or as antibacterial [22] or antiviral agents [23]. With a total change in FL of 20 ns between high and low $\mathrm{pH}$, acridine has potential to match, or even exceed, the accuracy of the electrochemical sensors, even when modest frequency-domain approaches are used for performing the lifetime measurement. However, similar to optical $\mathrm{pH}$ indicators in general, the $\mathrm{pH}$ sensing range for acridine is limited to 2-3 $\mathrm{pH}$ units centered on the $\mathrm{pK}_{\mathrm{a}}$ of the indicator, i.e. $\sim \mathrm{pH}$ 5.5-8 [18]. Therefore, it is necessary to find high response FL indicators across a range of $\mathrm{pK}_{\mathrm{a}}$ values. To date, no other organic FL $\mathrm{pH}$ indicators with a sensitivity which matches that of acridine has been proposed. Sensitive indicators that cover the sensing ranges below $\mathrm{pH}<6$ or $\mathrm{pH}>8$ are therefore required.

In previous work, it was shown that the $\mathrm{pK}_{\mathrm{a}}$ of acridine could be shifted to about 9 when immobilized in Nafion [18]. This highlights the importance when selecting the immobilizing medium, which should provide protection for the FL indicator molecule, without adversely influence the sensing characteristics. Mesoporous silica shows particular promise as an immobilizing medium due to high surface area offering potential high indicator loadings for increased signal, and good proton transport properties [20].

Here we present the $\mathrm{pH}$ induced FL response characteristic of two commercially available acridine derived fluorophores, 9-acridinemethanamine (9-AMA) and acridine-9carboxaldehyde (9-ACA), see Fig. 1, which we show are sensitive in the lower $\mathrm{pH}$ ranges from $\mathrm{pH} 2-6$. This $\mathrm{pH}$ range is relevant for both physiological applications and industrial applications such as those involving fermentation [24]. Furthermore, we assessed the characteristics of the indicators following immobilization in solid matrices. 9-AMA is immobilized in a surfactant phase within a mesoporous silica, whereas 9-ACA is bound covalently to amine-modified porous silica via the aldehyde group.

\section{Materials and Methods}

\section{Fluorescence Lifetime Indicators}

Acridine (97\%), (3-Aminopropyl) triethoxysilane (APTES, 98\%), tetraethoxysilane (TEOS, 98\%) and acridine-9carbaldehyde (9-ACA), tetradecyltrimethylammonium bromide (TTAB) was obtained from Sigma Aldrich. 9acridinemethanamine (9-AMA) was obtained from Fluorochem.

\section{Immobilization of 9-ACA in Amine Modified Silica Particles}

Amine modified silica particles were prepared as described by Chen et al. [25]. In short, TEOS and APTES were mixed in a 1:1 mol ratio. The samples were mixed at room temperature for one hour, after which the opaque suspensions were centrifuged to separate the particles and solution. The particles were washed three times in water and finally vacuum-dried overnight. The dried powders were subsequently suspended in

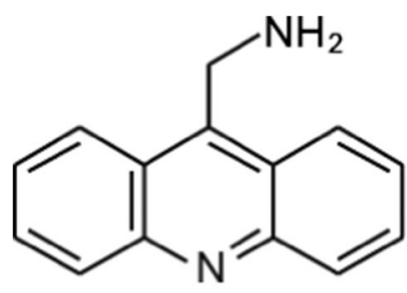

9-Acridinemethanamine (9-AMA)

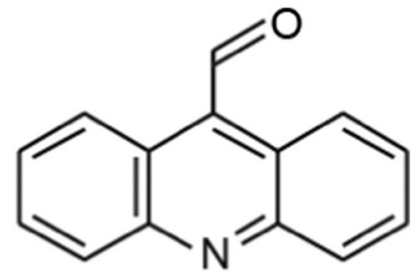

Acridine-9-carboxaldehyde (9-ACA)
Fig. 1 Chemical structures of 9-AMA and 9-ACA 
$10 \mathrm{~mL}$ of $10 \mathrm{mM}$ 9-ACA in methanol and mixed overnight. The final powder was washed twice with methanol and then once with water before vacuum-drying. The 9-ACA bonds covalently to primary amines via the aldehyde functional group, see Fig. 2.

\section{Immobilization of 9-AMA in Mesoporous Silica Particles}

9-AMA was immobilized according to a procedure we have published previously [20]. In short, a mesoporous silica was synthesized by mixing 2.3 mmoles of TTAB and 0.23 mmoles of 9-AMA in $16.7 \mathrm{~mL}$ water, which was gently heated to dissolve the powders completely. After cooling to room temperature, $25.3 \mathrm{~mL}$ EtOH and $4.4 \mathrm{~mL} \mathrm{NH}_{3}$ were added and the solution stirred for $15 \mathrm{~min}$. Then $1.66 \mathrm{~mL}$ TEOS was added and the solution stirred for an additional $2 \mathrm{~h}$. The powder was washed four times by shaking with $40 \mathrm{~mL}$ water followed by centrifugation to remove the water. The powder was finally dried at $50^{\circ}$ overnight. 9-AMA then remains immobilized within in the TTAB phase of the mesoporous network. There was observed to be negligible leaching of 9-AMA from the particles, rapid response times and very good proton transport properties. For a full characterization of this material, see reference [20].

\section{Fluorescence Lifetime Measurements}

In obtaining the FL of the immobilized acridine, fluorescence excitation was stimulated using sub-nanosecond pulses from a PicoQuant PLS light emitting diode with emission centered at $380 \mathrm{~nm}$. The resulting fluorescence was collected using a

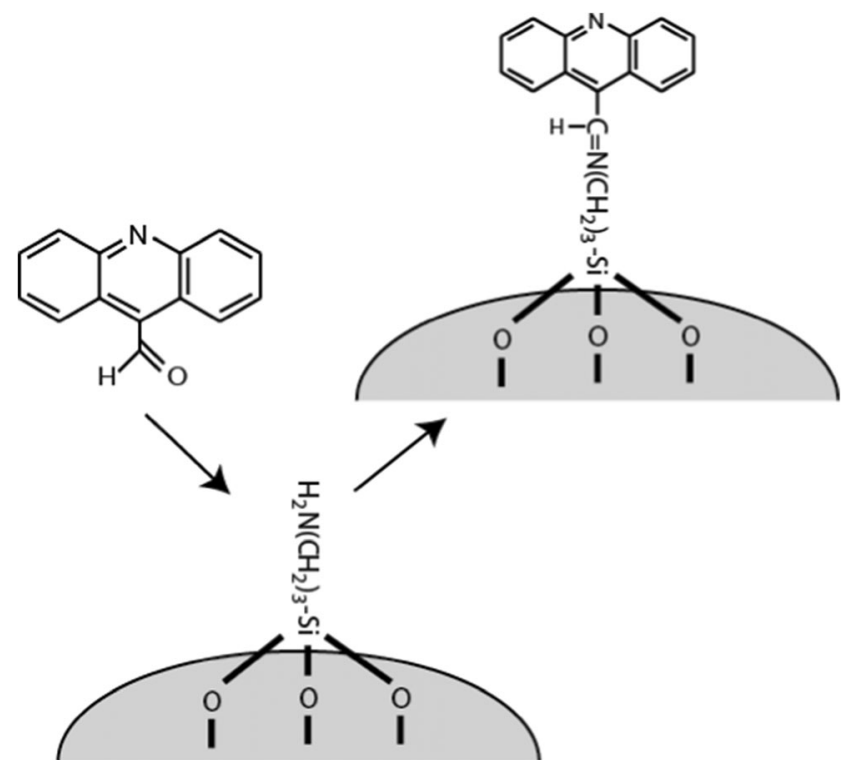

Fig. 2 Schematic description of the material synthesized by bonding 9 ACA to amine-modified porous silica
PicoQuant PMA 175 photomultiplier, fitted with a $45 \mathrm{~nm}$ spectral filter centered at $452 \mathrm{~nm}$. The photomultiplier response was digitized using a TimeHarp 260 Nano. The fluorescence lifetime values reported were determined by fitting exponential curves to the resulting fluorescence decay data using the FluoFit Pro software package.

\section{Fluorescence Emission Spectra}

The emission spectra were obtained by illuminating samples using a Thorlabs LED with emission centered at $370 \mathrm{~nm}$. The resulting fluorescence was collected and analyzed using a USB 2000 Ocean optics spectrometer, fitted with a $395 \mathrm{~nm}$ long pass filter.

\section{Results and Discussion}

\section{9-AMA}

Figure 3 shows FL versus $\mathrm{pH}$ for 9-AMA, both dissolved in $100 \mathrm{mM}$ phosphate buffer and incorporated into the palisade region of a surfactant-filled mesoporous silica. Immobilization in the surfactant template of mesoporous silica has been shown to provide a stable chemical environment for FL fluorophores, negligible leaching, good proton transport properties, and a high loading capacity for the hydrophobic fluorophore [20]. Similar materials have successfully been used to host chemo-sensing molecules in general [26, 27].

The $\mathrm{pK}_{\mathrm{a}}$ of 9 -AMA is predicted at $7.8(\mathrm{ACD} / \mathrm{Labs}$ Software). This is an expected value for the primary amine group of the molecule. However, the tertiary amine of 9AMA, which is an integral part of the aromatic rings, can be expected to have a lower $\mathrm{pK}_{\mathrm{a}}$. Thus, the $11 \mathrm{~ns}$ shift in FL between $\mathrm{pH} 2-5$ is likely related to the tertiary amine. In comparison, the tertiary amine of the parent molecule of 9AMA and 9-ACA, acridine, has a ground-state $\mathrm{pK}_{\mathrm{a}}$ of 5.45 in aqueous solution, and the protonated and neutral form of

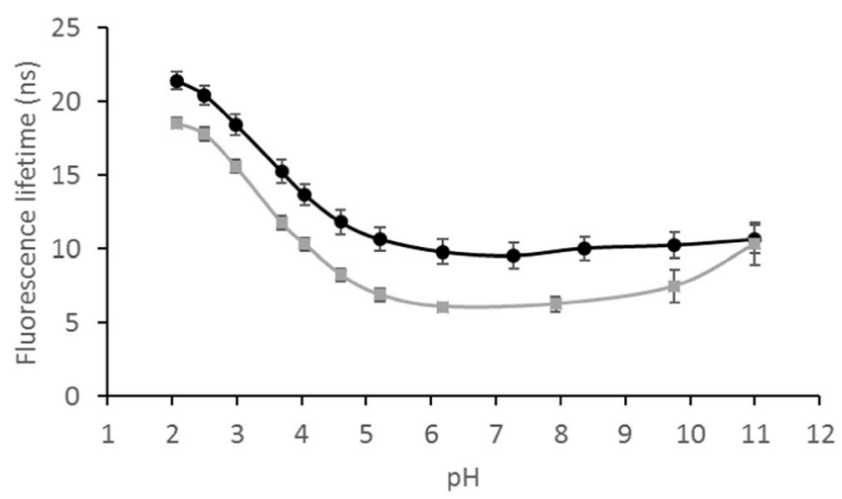

Fig. 3 The fluorescence lifetime of 9-AMA versus $\mathrm{pH}$ dissolved in $100 \mathrm{mM}$ buffer solutions $(\square)$ and immobilized in a surfactant-filled mesoporous silica $(\bullet)$ 
acridine has a FL of 31.1 and $6.6 \mathrm{~ns}$, respectively [18]. The increase in FL shows that the fraction of protonated 9-AMA increases sharply with a decrease in $\mathrm{pH}$ from 5 to 2 , indicating a $\mathrm{pK}_{\mathrm{a}}$ of around 3.5. Since the lone pair electrons of the tertiary amine occupy an $\mathrm{sp}^{2}$-orbital, conjugation with the $\pi$ electron system of the aromatic ring system is not possible; hence, they are readily accessible for protonation.

It is not surprising that protonation/deprotonation of the tertiary amine has a significant impact on the FL of 9-AMA, given the corresponding effect observed on the FL of acridine [18, 20]. The primary amine on the other hand, is located two bonds apart from the aromatic rings, and may as such not have a similar impact on the FL. At $\mathrm{pH}$ values above the expected $\mathrm{pK}_{\mathrm{a}}$ of the primary amine (7.8), an increase in FL is observed, which is most pronounced above $\mathrm{pH} 9.8$ where a 2.9 ns increase in FL occurs between $\mathrm{pH}$ 9.8-11. However, when the 9-AMA was incorporated into the surfactant phase of the mesoporous silica, the FL is nearly unchanged at $\mathrm{pH}$ values above 6 . This is apparently an effect of the immobilization, and can be due to the orientation of 9-AMA in the surfactant palisade layer. Should the primary amine for example be located deeper into the surfactant layer, it will be less accessible to protonation.

Figure 4 shows the recorded spectra of 9-AMA in solution (A) and immobilized (B), at both high and low $\mathrm{pH}$. In each case
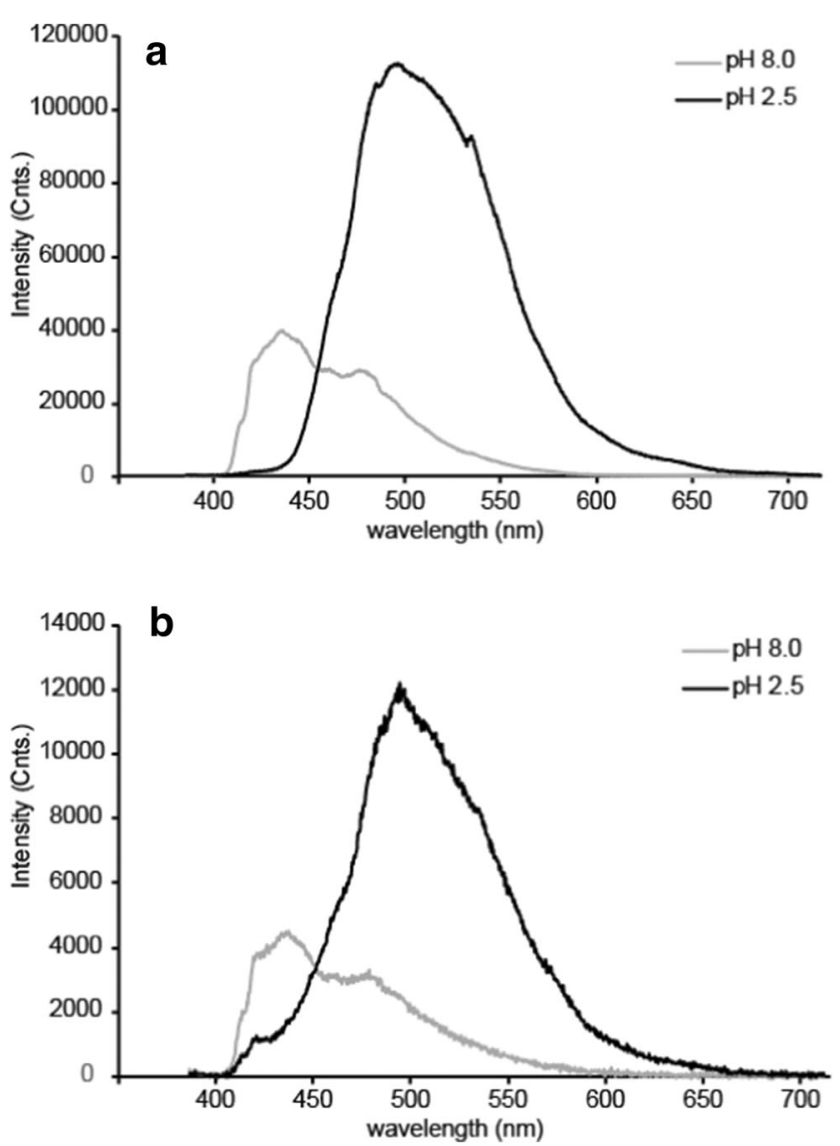

Fig. 4 Emission spectra of 9-AMA dissolved in buffer solutions (a) and immobilized in mesoporous silica (b), at $\mathrm{pH} 8$ and 2.5 there is both a shift in emission wavelength and a significant reduction in intensity on going from low to high $\mathrm{pH}$. At $500 \mathrm{~nm}$ emission, there is an $82 \%$ reduction in intensity from $\mathrm{pH} 2.5$ to 8.0. In the same $\mathrm{pH}$ interval, there is a shift in the peak emission wavelength of about $75 \mathrm{~nm}$. The red-shifted spectrum at low $\mathrm{pH}$ indicates presence of more resonance structures to stabilize the protonated molecule. Since the lone electron pair of the neutral tertiary amine belongs to an $\mathrm{sp}^{2}$ hybrid orbital, these do not contribute to the cyclic $\pi$-system and do not participate in resonance. This is likely the reason for this observation. In the neutral molecule, the $\mathrm{C}=\mathrm{N}$ bond functions as an effective electron sink, causing various resonance structures to occur due to the partial positive charges created about the carbons in the ring. When protonated, an additional resonance structure occurs, which shifts the spectrum to longer wavelengths.

The figure shows that at $450 \mathrm{~nm}$ the intensity contributions for both the long and short lifetime species are approximately equal, justifying the choice of optical filters used when acquiring the FL data. The relatively modest change in the FL and fluorescence response properties of 9-AMA following immobilization is most likely due to the electrostatic nature of the indicator-immobilizer interaction, leaving the indicator's internal electron transport properties relatively unchanged.

\section{9-ACA}

Figure 5 shows that $\mathrm{pH}$ has a negligible effect on the FL of 9ACA. This can be due to the $\pi$-electrons of the $\mathrm{C}=\mathrm{O}$ double bond interfering with those of the aromatic rings. However, when bound to the amine modified silica, the $\mathrm{pH}$ response characteristics changed dramatically, with the material exhibiting a FL decrease of $15 \mathrm{~ns}$ with increasing $\mathrm{pH}$ through the range $\mathrm{pH} 3-6$.

Hence, in contrast to 9-AMA, the fluorescence spectrum of 9ACA differs significantly for the buffer solubilized and immobilized cases (see Fig. 6). Immobilized 9-ACA exhibits a spectral shift to longer wavelengths with decreasing $\mathrm{pH}$, similar to 9-AMA, whereas the solubilized 9-ACA shows no spectral shift. This indicates that the resonance structure attributed to the

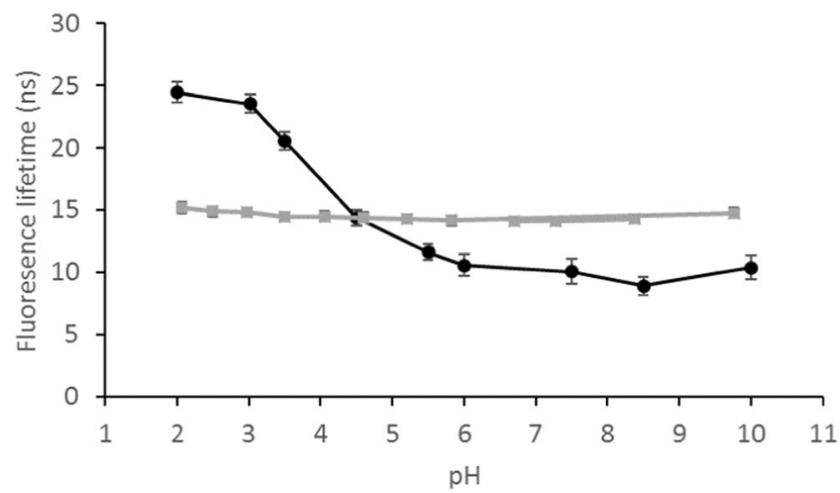

Fig. 5 The fluorescence lifetime of 9-ACA versus $\mathrm{pH}$ dissolved in $100 \mathrm{mM}$ buffer solutions ( $\square$ ) and covalently bound to amine-modified silica $(\bullet)$ 
red-shifted spectrum at low $\mathrm{pH}$ does not occur in the buffer stabilized molecule, and it seems this resonance form is crucial for obtaining the good FL pH sensitivity. The dramatic change in the $\mathrm{pH}$ response behavior of both the fluorescence intensity spectrum and the FL for 9-ACA following immobilization is attributed to the effects of the 9-ACA - silica covalent bonds (see Fig. $2)$. During the process of surface bonding, the carbonyl $(\mathrm{C}=\mathrm{O})$ group adjacent to the aromatic rings is substituted with the less polar imine $(\mathrm{C}=\mathrm{N})$ group (Fig. 2). Such an alteration in polarity could explain the observed difference between the fluorescence properties of the surface bonded and buffer solubilized species. Moreover, imines are themselves mildly basic and will protonate at low $\mathrm{pH}$. Thus, it is possible that the imine protonation contributes to the $\mathrm{pH}$ sensitivity of immobilized fluorophore fluorescence lifetime.

An advantage with this material is that the indicator is covalently bonded to the immobilizing matrix. This is convenient when considering long-term stability in a compact optical sensor, due to absence of e.g. the indicator leaching from or migrating within the immobilizing material.

Several fluorescence-based $\mathrm{pH}$ sensing principles have been proposed, where most rely on a change in intensity. This is caused by protonation/deprotonation of functional groups in close vicinity
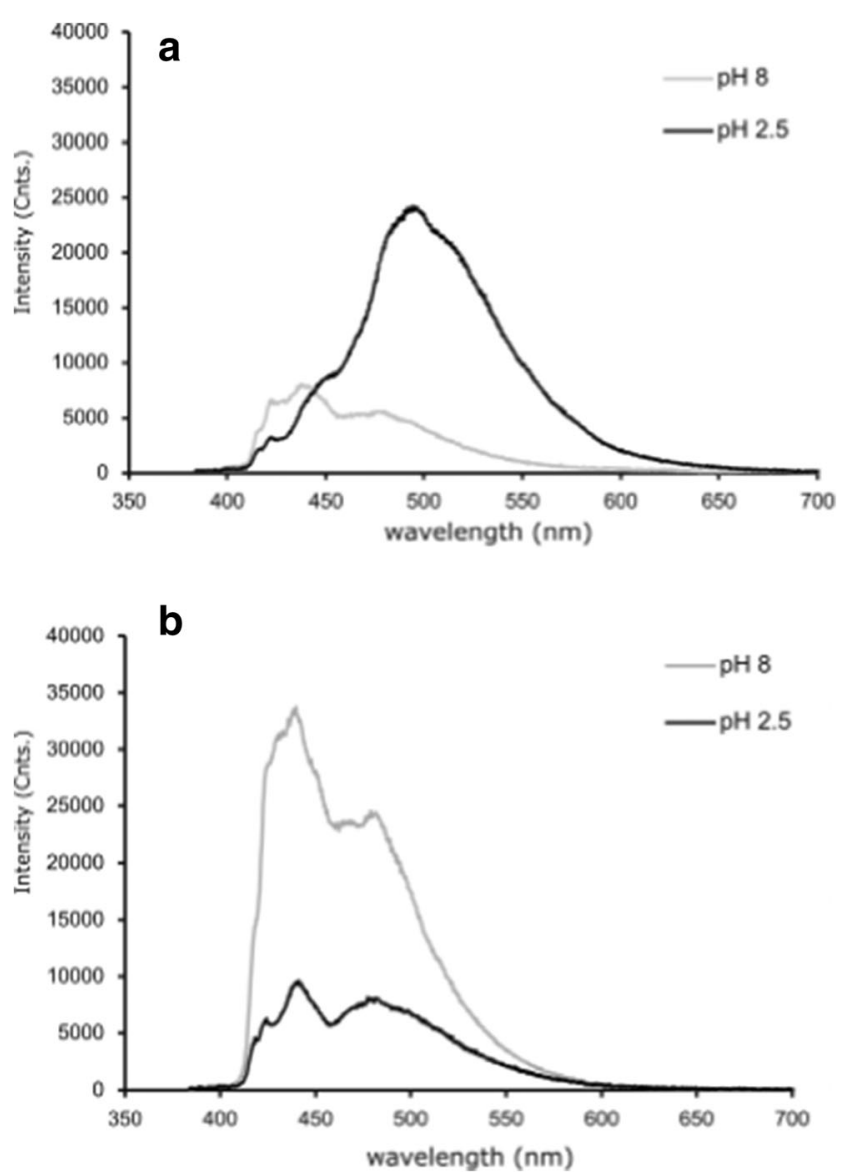

Fig. 6 Emission spectra of 9-ACA covalently bound to silica (a) and dissolved in buffer solutions (b) and, at $\mathrm{pH} 8$ and 2.5 to the aromatic rings, which leads to partial quenching of the fluorescence. As mentioned, the motivation for using FL rather than intensity to monitor $\mathrm{pH}$ is that $\mathrm{FL}$ is an intrinsic property of an indicator, which is not affected by e.g. leaching, light scattering effects, excitation source intensity variations or photobleaching. Unfortunately, although many fluorophores have variations in fluorescence intensity with changes in $\mathrm{pH}$, very few of these show significant FL pH sensitivity.

Acridine is amongst the indicators with highest fluorescence lifetime responses to $\mathrm{pH}[18-20]$, in the range of $\sim \mathrm{pH}$ 5.5-8. Acridine immobilized in Nafion has previously been shown to be sensitive in the $\mathrm{pH}$ range between about 8.5-10.5 [18]. Thus, a higher $\mathrm{pH}$ FL indicator candidate to those presented here is available, although the important sensing range for oceanographic applications, between $\mathrm{pH} 8-8.5$, remains a blind spot in terms of sensitive FL indicators. A previous publication indicated that diethylaminomethyl pyrene has potential as a sensitive fluorescence lifetime $\mathrm{pH}$ indicator in this $\mathrm{pH}$ range [28]. However, a major drawback with pyrene derived FL indicators is their high sensitivity to oxygen [29].

We recently showed that the FL $\mathrm{pH}$ sensing range of acridine can be extended by placing the fluorophore in a $\mathrm{pH}$ sensitive chemical environment, in this case an amine modified porous silica [12]. However, the total change in FL remains similar, and in order to achieve the highest possible sensitivity, it is an advantage to apply several sensitive indicators with different sensing ranges.

\section{Conclusions}

9-ACA and 9-AMA complement acridine in widening the $\mathrm{pH}$ range where sensitive fluorescence lifetime indicators are available. These three indicators have overlapping sensing ranges from $\mathrm{pH} 2-8$, which covers a broad range of applications. The $\mathrm{pK}_{\mathrm{a}}$ of 9-AMA and immobilized 9-ACA lie at approximately 3.5 , which to the best of our knowledge is lower than all other FL $\mathrm{pH}$ sensing fluorophores that have been demonstrated previously. With respect to the applicability of 9-ACA and 9-AMA as potential indicators in compact optical sensors, the long lifetime of these fluorophores makes it easier to delineate the signal from background fluorescence, which can be a considerable limitation for the application of fluorescence-based $\mathrm{pH}$ sensing [24]. Furthermore, we have demonstrated that both 9-ACA and 9AMA can be successfully immobilized in robust substrates. Finally, the large FL shifts observed with $\mathrm{pH}$, enables relatively simple optoelectronics to be used for their determination.

Acknowledgements The present study was supported by the Research Council of Norway (grant number 269090), and by Aanderaa - a Xylem brand. 
Funding Information Open Access funding provided by Norwegian Geotechnical Institute

Open Access This article is licensed under a Creative Commons Attribution 4.0 International License, which permits use, sharing, adaptation, distribution and reproduction in any medium or format, as long as you give appropriate credit to the original author(s) and the source, provide a link to the Creative Commons licence, and indicate if changes were made. The images or other third party material in this article are included in the article's Creative Commons licence, unless indicated otherwise in a credit line to the material. If material is not included in the article's Creative Commons licence and your intended use is not permitted by statutory regulation or exceeds the permitted use, you will need to obtain permission directly from the copyright holder. To view a copy of this licence, visit http://creativecommons.org/licenses/by/4.0/.

\section{References}

1. Narayanaswamy R, Wolfbeis O (2013) Optical sensors: industrial environmental and diagnostic applications, vol 1. Springer Science \& Business Media

2. Atamanchuk D, Kononets M, Thomas PJ, Hovdenes J, Tengberg A, Hall PO (2015) Continuous long-term observations of the carbonate system dynamics in the water column of a temperate fjord. J Mar Syst 148:272-284. https://doi.org/10.1016/j.jmarsys.2015.03.002

3. Thomas PJ, Atamanchuk D, Hovdenes J, Tengberg A (2017) The use of novel optode sensor technologies for monitoring dissolved carbon dioxide and ammonia concentrations under live haul conditions. Aquac Eng 77:89-96. https://doi.org/10.1016/j.aquaeng. 2017.02.004

4. Damborský P, Švitel J, Katrlík J (2016) Optical biosensors. Essays Biochem 60:91-100. https://doi.org/10.1042/EBC20150010

5. Wencel D, Abel T, McDonagh C (2014) Optical chemical pH sensors. Anal Chem 86:15-29. https://doi.org/10.1021/ac4035168

6. Liebsch G, Klimant I, Krause C, Wolfbeis OS (2001) Fluorescent imaging of $\mathrm{pH}$ with optical sensors using time domain dual lifetime referencing. Anal Chem 73:4354-4363. https://doi.org/10.1021/ ac0100852

7. Borisov SM, Gatterer K, Klimant I (2010) Red light-excitable dual lifetime referenced optical $\mathrm{pH}$ sensors with intrinsic temperature compensation. Analyst 135:1711-1717. https://doi.org/10.1039/C0AN00180E

8. Staudinger C, Strobl M, Breininger J, Klimant I, Borisov SM (2019) Fast and stable optical $\mathrm{pH}$ sensor materials for oceanographic applications. Sensors Actuators B Chem 282:204-217. https:// doi.org/10.1016/j.snb.2018.11.048

9. Meier RJ, Simburger JMB, Soukka T, Schaferling MA (2015) FRET based $\mathrm{pH}$ probe with a broad working range applicable to referenced ratiometric dual wavelength and luminescence lifetime read out. Chem Commun 51:6145-6148. https://doi.org/10.1039/ C5CC00144G

10. Hynes J, O'Riordan TC, Zhdanov AV, Uray G, Will Y, Papkovsky DB (2009) In vitro analysis of cell metabolism using a long-decay $\mathrm{pH}-$ sensitive lanthanide probe and extracellular acidification assay. Anal Biochem 390:21-28. https://doi.org/10.1016/j.ab.2009.04.016

11. Turel M, Čajlaković M, Austin E, Dakin JP, Uray G, Lobnik A (2008) Direct UV-LED lifetime $\mathrm{pH}$ sensor based on a semipermeable sol-gel membrane immobilized luminescent Eu3+ chelate complex. Sensors Actuators B Chem 131:247-253. https://doi. org/10.1016/j.snb.2007.11.047

12. Totland C, Thomas PJ, Holst B, Akhtar N, Hovdenes J, Skodvin T (2019) A broad-range fluorescence lifetime $\mathrm{pH}$ sensing material based on a single organic fluorophore. J Fluorescence 9:11251131. https://doi.org/10.1007/s10895-019-02426-9
13. Berezin MY, Achilefu S (2010) Fluorescence lifetime measurements and biological imaging. Chem Rev 110:2641-2684. https:// doi.org/10.1021/cr900343z

14. Berezin MY, Guo K, Akers W, Northdurft RE, Culver JP, Teng B, Vasalatiy O, Barbacow K, Gandjbakhche A, Griffiths GL, Achilefu S (2011) Near-infrared fluorescence lifetime $\mathrm{pH}$-sensitive probes. Biophys J 100:2063-2072. https://doi.org/10.1016/j.bpj.2011.02.050

15. Orte A, Alvarez-Pez JM, Ruedas-Rama MJ (2013) Fluorescence lifetime imaging microscopy for the detection of intracellular $\mathrm{pH}$ with quantum dot nanosensors. ACS Nano 7:6387-6395. https:// doi.org/10.1021/nn402581q

16. Zheng C, An X, Gong J (2015) Novel pH sensitive N-doped carbon dots with both long fluorescence lifetime and high quantum yield. RSC Adv 5:32319-32322. https://doi.org/10.1039/C5RA01986A

17. Dalfen I, Dmitriev RI, Holst G, Klimant I, Borisov SM (2018) Background-free fluorescence-decay-time sensing and imaging of $\mathrm{pH}$ with highly photostable diazaoxotriangulenium dyes. Anal Chem 91:808-816. https://doi.org/10.1021/acs.analchem.8b02534

18. Ryder AG, Power S, Glynn TJ (2003) Evaluation of Acridine in Nafion as a fluorescence-lifetime-based $\mathrm{pH}$ sensor. Appl Spectrosc 57:73-79. https://doi.org/10.1366/000370203321165232

19. Ryder AG, Power S, Glynn TJ, Morrison JJ (2001) Time-domain measurement of fluorescence lifetime variation with $\mathrm{pH}$. Proc SPIE 4259. https://doi.org/10.1117/12.432487

20. Totland C, Thomas PJ, Holst B, Akhtar N, Hovdenes J, Skodvin T (2019) The use of surfactant-filled mesoporous silica as an immobilising medium for a fluorescence lifetime $\mathrm{pH}$ indicator, providing long-term calibration stability. RSC Adv 9:37241-37244. https://doi.org/10.1039/C9RA07374D

21. Philippe B, Johann B, Thomas G, Martin T (2007) Acridine and Acridone derivatives, anticancer properties and synthetic methods: where are we now? Anti Cancer Agents Med Chem 7:139-169. https://doi.org/10.2174/187152007780058669

22. Wainwright $\mathrm{M}$ (2001) Acridine - a neglected antibacterial chromophore. J Antimicrob Chemother 47:1-13. https://doi.org/10.1093/jac/47.1.1

23. Bastow K (2004) New Acridone inhibitors of human herpes virus replication. Curr Drug Targets Infect Disord 4:323-330. https://doi. org/10.2174/1568005043340533

24. Janzen NH, Schmidt M, Krause C, Weuster-Botz D (2015) Evaluation of fluorimetric $\mathrm{pH}$ sensors for bioprocess monitoring at low pH. Bioprocess Biosyst Eng 38:1685-1692. https://doi. org/10.1007/s00449-015-1409-4

25. Chen S, Hayakawa S, Shirosaki Y, Fujii E, Kawabata K, Tsuru K, Osaka A (2009) Sol-gel synthesis and microstructure analysis of amino-modified hybrid silica nanoparticles from aminopropyltriethoxysilane and tetraethoxysilane. J Am Ceram Soc 92:2074-2082. https://doi.org/10.1111/j.1551-2916.2009. 03135.x

26. Onida B, Bonelli B, Flora L, Geobaldo F, Arean CO, Garrone E (2001) Permeability of micelles in surfactant-containing MCM-41 silica as monitored by embedded dye molecules. Chem Commun 2216-2217. https://doi.org/10.1039/B105261F

27. Onida B, Borello L, Fiorilli S, Bonelli B, Arean CO, Garrone E (2004) Mesostructured SBA-3 silica containing Reichardt's dye as an optical ammonia sensor. Chem Commun:2496-2497. https:// doi.org/10.1039/B409779C

28. Draxler S, Lippitsch ME (1995) pH sensors using fluorescence decay time. Sensors Actuators B Chem 29:199-203. https://doi. org/10.1016/0925-4005(95)01683-X

29. Draxler S, Lippitsch ME (1996) Lifetime-based sensing: influence of the microenvironment. Anal Chem 68:753-757. https://doi.org/ $10.1021 / \mathrm{ac} 9507092$

Publisher's Note Springer Nature remains neutral with regard to jurisdictional claims in published maps and institutional affiliations. 